\title{
AHP, ANP, AND ANN: TECHNICAL DIFFERENCES, CONCEPTUAL CONNECTIONS, HYB RID MODELS
}

\author{
ISPRI (Institute for Forecasting Studies and International Research) \\ info@ispri.org \\ CSSI (University Centre of International and Strategic Studies) \\ presidente@cssi.unifi.it
}

AUTHORS:

Sergio Bedessi

ISPRI

Florence, Italy

info@ ispri.org*

Serena Lisi

CSSI

Florence, Italy

web.editor@cssi.unifi.it*

\begin{abstract}
The AHP (Analytic Hierarchy Process) and the ANP (Analytic Network Process) are formalised decision making methods that examine quantitative and qualitative factors, while ANN (Artificial Neural Networks) can be considered to be data processing systems that are based on how the human brain works, and operate by assigning weights as does a universal function approximator. AHP and ANP are based on an "objective-criteria-alternative" structure; AHP operates hierarchically, while the evolution of this model, ANP, introduces the concepts of feedback and interdependence. In this sense, ANP may be seen as a type of "conceptual bridge" between AHP and ANN (when taking into consideration those neural networks that learn through the so-called backpropagation algorithm). This study presents an AHP-ANN hybrid model in which AHP is used during the decision making phase and one or more ANNs substitute the classic pair-wise comparisons typical of AHP assigning weights to the alternatives with regard to one or more of the criteria. Moreover, the study attempts to define the parameters and the case records for which the model is applicable, so as to propose its generalised use rather than ad hoc use for specific cases and situations.
\end{abstract}

\section{Introduction}

The AHP (Analytic Hierarchy Process) and the ANP (Analytic Network Process) are formalised methods of decision making that are capable of taking quantitative factors into consideration alongside the qualitative factors. AHP may specifically be described as a general method used to represent problems that have an hierarchical structure, which defines each problem in terms of

\footnotetext{
"Under the supervision of Prof. Umberto Gori, Emeritus, Political Science Faculty "Cesare Alfieri", University of Florence, Italy.

The English translation has been provided by Margot J.Wylie, CSSI

*Under the supervision of Prof. Umberto Gori, Emeritus, Political Science Faculty "Cesare Alfieri", University of Florence, Italy.

The English translation has been provided by Margot J.Wylie, CSSI
} 
objectives, criteria and alternatives, while ANP (the evolution of AHP), through the introduction of the concepts of interdependence and feedback into the model, is able to represent problems in which the importance of the alternatives may in turn modify the importance attributed to the criteria (Saaty, 2003).

This study aims to demonstrate how AHP, when applied to certain cases or in relation to single situations, may be significantly improved by building a hybrid model with one or more ANNs.

Several preliminary remarks must be made regarding the techniques that are characteristic of ANNs. Artificial neural networks may be considered to be a system for processing information modelled after how the human brain works; these artificial networks, however, do not reproduce the exact structure of the human brain insofar as this last is extremely complex. The human mind, in fact, is the home of various types of thought processes, be they rational, formalised, and conscious, be they less rational and formalised but still conscious, or be they thought processes that are not rational, formalised or conscious. Artificial neural networks to some degree reproduce the non-formalised thought that originates from the thick web of neurons that make up the cerebral mass.

The various types of artificial neural networks are successfully used to make forecasts in a wide range of fields (financial and economic, tourism, meteorology, security, and so forth) and, with respect to more classic information processing systems that are based on heteronomous serial logic, ${ }^{1}$ offer the advantage that they are capable of learning from their own experiences.

From a forecasting point of view, several types of $\mathrm{ANN}^{2}$ behave like universal function approximators, with the benefit that they are able to obtain excellent results even with non-linear functions; they may also be seen as a black-box, in the sense that they are able to autonomously find the function that connects independent variables to dependent variables.

\section{Conceptual connections and technical differences}

Given the characteristics of the three techniques, it is possible to attempt a conceptual and structural comparison that focuses particularly on AHP and ANN; among the many possible analogies, the common concept of creating decision making "nodes" emerges, and may be understood to be much like the structures typical of the interactions occurring within the human brain. Thanks to this concept, it is possible to lay the foundation for structuring a hybrid system that is able to capitalise on and optimise the potential uses of the two techniques when applied to specific and complex situations. The situations in question may be defined as "particular", not in relation to the specific area of use, but rather because the model, in order to be applied, must be applied according to well-defined characteristics, which will be described in section 2. In this context, ANP, given the presence of networks, may be considered to be an intermediate step, a type of "conceptual bridge" that unites the underlying rationale between AHP and ANN. In fact, both ANP and AHP divide the decision into the same components (objective, criteria, alternatives, inserted into structures that may be defined as clusters), and both are based on the comparison between couples. ANP, on the other hand, is able to establish transverse connections that, thanks to the concepts of interdependence and feedback, create a network-like structure similar to that used by the ANN.

Although ANP constitutes a conceptual "bridge" between AHP and ANN, important technical differences must be taken into consideration beginning with the mathematical structure that characterises the two theories. As aforementioned, AHP has an hierarchical structure that is connected to the structure of the decision that is to be taken and that may be described in terms of the objective, criteria (including eventual sub-criteria), and alternatives and is characterised by the system of pair-

1 Based on heteronomous serial logic (John Von Neumann's logic is in contrast with Norbert Wiener's autonomous logic), where the computer is an algorithmic executant that works on information, the meaning of which is only known to the person who inserted it.

2 The networks work thanks to the supervised learning paradigm. With an opportune training set at their disposal, these networks are trained using a back propagation algorithm that is just as important, which allows the parameters to be reviewed and modified according to the known data so as to minimise forecasting errors connected to the given training set. 
wise comparisons. This last allows for elements that are not normally measurable to be evaluated and is the necessary condition for which the system works. In the ANN, on the other hand, the internal structure in no way represents the structure of the problem to be analysed. However, while the ANN are capable of learning from their experiences, AHP always necessitates human evaluations. Although the systems that are used in the calculation and attribution of the position (or weight) differ, it is, at any rate, possible to conclude that all three structures (AHP, ANP, ANN) may be considered to be systems that generate a ranking by choice among the alternatives.

\section{A hybrid model}

It was the analysis of the characteristics of the two systems that lead to the proposal of creating a hybrid model where AHP is used in the decision making phase while one or more ANNs are used to assign the weight of the alternatives with regard to one or more of the criteria instead of the classic pair-wise comparisons typical of AHP.

This hypothesis has already been proposed by other research groups, but different methods were applied (Atzeni, Sanna, Spanu, 2006; Kauko, 2002; Kayikci, 2010; Kumar and Roy, 2010; Özdağoğlu and Özdağoğlu, 2007; Saeedi, Malek, Delavar and Tayybi, 2008; Wang, Huang, Nguyen and Krishnakumar, 2008). The aspect that sets this study apart from the rest resides in the fact that the hybrid system has been structured using the conceptual associations discussed above as a foundation, where AHP is allocated to the high level function of formalised reasoning (in particular to the decision making) and the ANN are used to perform lower level functions of non-formalised reasoning. Moreover, the introduction of a generalised use of the proposed model (that would be applicable to various disciplines and not only specific ad hoc cases) is attempted, even in association with other techniques wherein, depending on the case, the pair-wise comparisons are either completely or only partially substituted for the ANN.

Therefore, in general terms, it is possible to affirm that the model appears to be particularly promising in those cases where the choice of alternatives is not merely anchored to assessments connected to preferences but has to be based, at least in part, on data that are at that moment unknown yet are foreseeable because of the presence of temporal or spatial (or other) series. In these cases, the pairwise comparisons between the alternatives with regard to a specific criteria is substituted by the generation of a weight of that particular alternative compared to the criteria, a weight (dependent variable) that is generated by the ANN and that uses a series of independent variables for making the forecast.

See the diagram below:

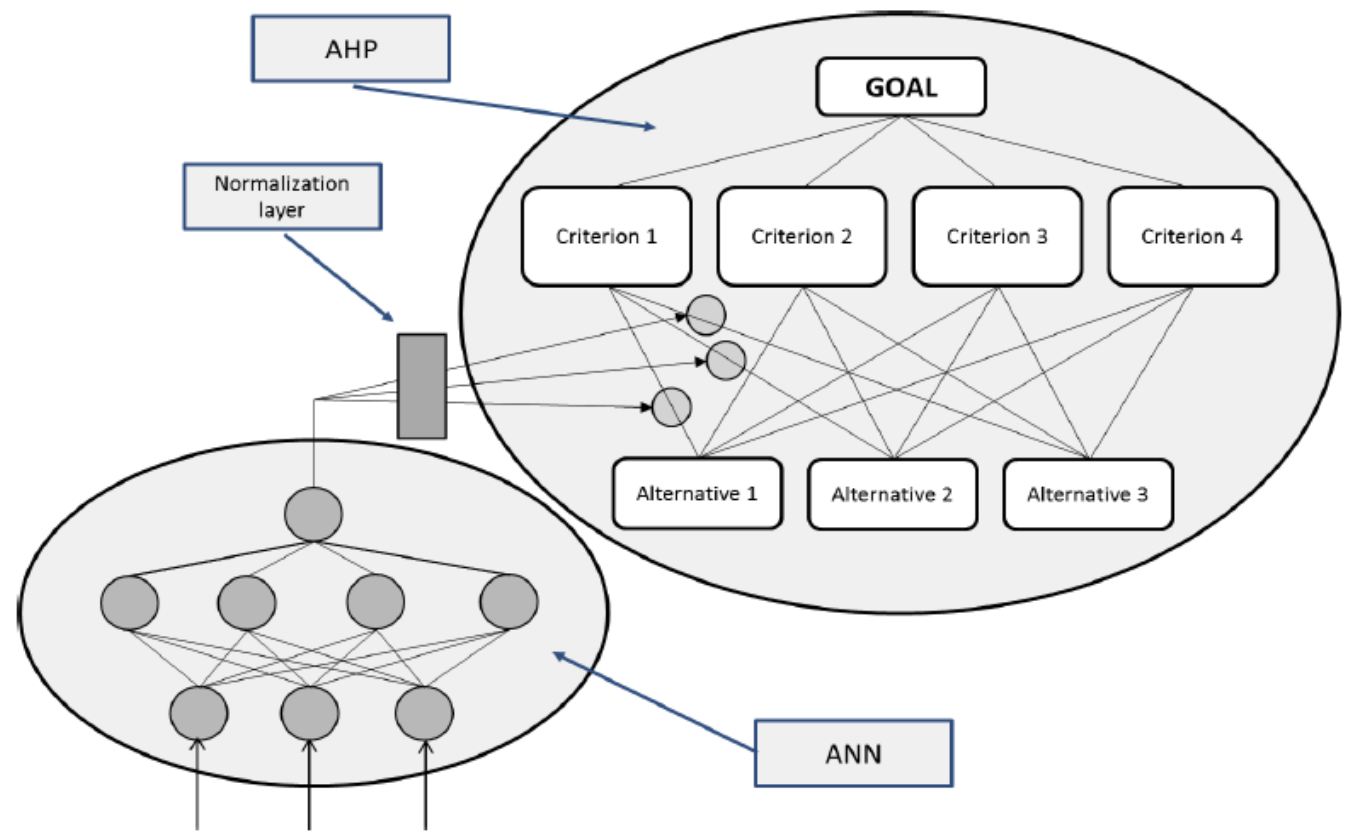

Figure 1. Hybrid model diagram. 
At first glance, the hybrid model might appear to trigger such extreme considerations as follows:

- some might believe that it is suitable only in specific instances for specific cases;

- some might consider it to be suitable for all types of decisions.

As was mentioned above, however, the model is suited to various types of decisions that exhibit certain characteristics, inasmuch as it was designed to use one (or more) ANN to attribute the weight of one, several, or all of the alternatives according to the cases examined, while for the evaluation of the various criteria (the one versus the others, to create the contingency table) the data would continue to be calculated using the pair-wise comparisons so characteristic of AHP.

A more extensive generalisation of the hybrid system could even go so far as to require that the weights assigned to the various criteria be generated by the artificial neural networks.

Obviously, the interconnection between the two systems, whichever the level that is ultimately implemented, needs a specific normalisation, insofar as the estimations are expressed in different bases (exiting values from 0 to 1 for the ANN, and, usually a range between 1 and 9 for the pair-wise comparisons in AHP) ${ }^{3}$.

In order for the model to work, the following conditions must be met:

- it is important that the case being considered doesn't just use simple preferences, or decisions that are to be taken on the basis of criteria that have been chosen beforehand, but also past experiences or forecasts based on previous data (not necessarily temporal in nature). In fact, in situations where forecasts based on series of data (temporal and not) are not assumed, the hybrid system risks falling into circular reasoning, or rather, in the fact that the past experiences of the training set could be chosen after the criteria had been laid out, which could result in the final choice being influenced and not entirely objective;

- it is important that a training set is built that is coherent with AHP's formalised reasoning; in this sense, even the data of the training set for the ANN have to refer back to a hypothetical unity understood to be $100 \%$ (the entirety of the sample). The transformation of the values coming out of the ANN in numbers that are significant for AHP is not a problem, as long as an appropriate algorithm is used;

- at any rate, the aim must be univocal, just like when the traditional AHP is used: the hybrid system, in fact, allows the evaluation of the alternatives with regard to an objective and not with multiple objectives.

\section{Advantages}

The advantage of using a hybrid model that employs one or more ANN resides in the fact that it is possible to apply one or more of the most powerful formalised decision making instruments, AHP, to situations in which pair-wise comparisons typical of the AHP could prove to be subjective or uncertain. In this way, the hybrid system would allow for the assessment of future developments of several situations related to the choice that would be made using the AHP decision making instrument, for example, the future political stability and the future exchange rate.

The hybrid system particularly lends itself to the use of AHP in making economic, political, and international security forecasts as well as in all those cases in which the choice of one of the alternatives is affected by parameters that are not definite but forecasts.

\section{Possibile applications}

Keeping in mind all that has been said thus far, some of the possible applications could be as follows:

3 A simple normalising algorith $\mathrm{m}$, considering that the sum of the weights of the alternatives with regard to a certain criterion must be equal to 1 , could be as follows: Given $w_{1}, w_{2}, w_{3}$ the weights of the alternatives 1,2 and 3, as they were identified by the $\mathrm{ANN}$, and $\mathrm{Sw}$ is the sum of the values of these weights, the weight to apply to the AHP system for the same alternatives with regard to the criterion already considered will be equal to $\mathrm{w}_{1}{ }^{\prime}=\mathrm{w}_{1} / \mathrm{Sw}$. Obviously, it would be possible to create different systems in the eventuality that one wanted to avoid a normalisation that is based on the proportion of the weights derived using the ANN with regard to their sum. 
- decision making processes in the tourism sector (such as choosing the location, from several alternatives, of a hotel using past data and applying one or more ANNs to forecast probable future socio-economic data to arrive at the best alternative, or choosing which is the most convenient marketing plan by creating a projection of the future socio-economic data);

- decision making processes in areas of security (for example, deciding which are the best outposts in war zones using AHP and one or more ANNs that, taking geographic data from past attacks, are able to project the risks of future attacks thus leading AHP to identify the best alternative position or positions);

- evaluating whether to participate in a multinational inter-service military operation or not. While AHP would perform the decision making, one or more ANNs could provide data for producing the assessment of alternatives with regard to particularly critical criteria (for example: the evolution of the politica situation, the evolution of the existing equilibrium among the insurgent groups);

- strategic decision making processes in a political, economic, social context (for example, decide whether to sign a certain type of treaty or not, or whether to participate in common polic ies, such as agricultural or monetary policies or policies in cooperation), where the assessment of several alternatives with regard to certain criteria could be facilitated by a system that is able to learn from data extracted from the past;

- the choice of a foreign country in which to outsource production using an ANN to provide the future parameters of political stability to AHP, along with other parameters necessary to making the assessment (ability of the available workforce, technical level of production, compatibility with company procedures with the conditions in the prospective country, exchange rate between the currencies of the two countries, cost of labour, ease of interaction with the unions, geographic proximity, tax breaks).

\section{Conclusions}

The possibility to use an AHP-ANN hybrid system ought to be seen in light of the need to apply the decision making process to situations in which the assessment of the alternatives already aims at taking future scenarios into consideration rather than actual data; the system, therefore, seems to be promis ing with regard to specific areas where the final decision must be taken using parameters and indicators that are, at first glance, uncertain (for example: the political stability, the risk level of a specific area in a territory, the possibility that a financial market will crash, and so on), but that are foreseeable using ANN.

Selecting the so called "training set" (which will be created using specific indicators) is undoubtedly of utmost importance, particularly for the areas of economic and political forecasting. It is the training set that the ANN will use to project values toward the AHP as well as the criteria for the assessment. This will require research analysis of the reliability of the sources, not so much regarding their statistical precision but rather the gathering of the data that will be examined. The indices for constructing the indicators as well as the methods to operationalise these last will be kept in mind with the aim to build a framework of data that is useful to the ANN serving the AHP system.

Lastly, the hybridisation presented in this paper has the potential to be expounded upon and could (all the while maintaing the connection to ANN) continue to evolve with the use of other techniques used in creating the weights of the alternatives with regard to the criteria (fuzzy logic, Lotka-Volterra equations, genetic algorithms). 


\section{REFERENCES}

Atzeni, C.; Sanna, U.; Spanu, N. (2006). “Applicazioni della matematica fuzzy per la selezione dei progetti conservativi nei siti archeologici". Archeologia e Calcolatori. 17, 2006. pp. 83-94. http://soi.cnr.it/archcalc/indice/PDF17/05_Atzeni.pdf

Bao, J. (2002, December). "Hierarchical Learning in Neural Network" A research notes/proposal. December 2002. http://www.cs.iastate.edu/ baojie/acad/current/hnn/hnn.htm

Galushkin, A. I. (2007). Neural Networks Theory. Springer-Verlag Berlin and Heidelberg GmbH \& Co.

Hassoun, M. H. (1995). Fundamentals of Artificial Neural Networks. MITPress.

Kauko, Tom Johannes. (2002). Modelling the locational determinants of house prices: neural network and value tree approaches. Faculteit Ruimte lijke Wetenschappen, Universiteit Utrecht, Utrecht.

Kayikci, Y. (2010). "A conceptual model for intermodal freight logistics centre location decisions". The Sixth International Conference on City Logistics. Procedia Social and Behavioral Sciences. 2 (2010) 6297-6311.

Kumar, J. and Roy, N. (2010, December) "A Hybrid Method for Vendor Selection using Neural Network". International Journal of Computer Applications (0975-8887). Vol. 11, No. 12. http://www.ijcaonline.org/volume11/number12/pxc3872193.pdf

Özdağoğlu, A. and Özdağoğlu, G (2007). "Comparison of AHP and Fuzzy AHP for the Multicriteria Decision Making Processes with Linguistic Evaluations". İstanbul Ticaret Üniversitesi Fen Bilimleri Dergisi Yll: 6 Say1:11Bahar 2007/1 s. 65-85.

Peretto, P. (1994). An Introduction to the Modeling of Neural Networks. Collection Alea-Saclay, Monographs and Texts in Statistical Physics, Cambridge University Press.

Saeedi, S.; M. Malek; M. Delavar; A. Tayybi. (2008, June 14-16). Intuitionistic Fuzzy Analytical Network Process (IF-ANP) for Spatial Multi Criteria Decision Making under Uncertainty. Integrating Generations. FIG Working Week 2008. Stockholm, Sweden.

Saaty, R. W. (2003). Decision Making in Complex Environments - The Analytic Hierarchy Process (AHP) for Decision Making and The Analytic Network Process (ANP) for Decision Making with Dependence and Feedback. Superdecisions Tutorial.

Wang, X.; Huang, Y.; Nguyen, N. and Krishnakumar, K. (2008). "CBN tool flank wear modelling using Hybrid Neural Network”. Int. J. Mechatronics and Manufacturing Systems, Vol. 1, No. 1, pp. 83102.

Yegnanarayana, B. (2004). Artificial Neural Networks. Prentice-Hall of India. 